\title{
STRONGYLOSES GASTRO-INTESTINALES ET MALABSORPTION DES NUTRIMENTS
}

\author{
A. DAKKAK
}

\begin{abstract}
RÉSUMÉ
Strongyloses gastro-intestinales et absorption des nutriments.

Le rôle de la malabsorption des nutriments dans le mécanisme de la diminution des productions des ruminants infestés par des nématodes parasites du tractus gastro-intestinal a fait l'objet d'une controverse à cause de l'absorption compensatrice et de la réabsorption qui se développent en aval du site de l'infestation. Au
\end{abstract}

cours de la dernière décennie, des recherches approfondies lui attribuent, cependant, de plus en plus un rôle important dans ce mécanisme car : 1) l'absorption compensatrice et la réabsorption n'intéressent pas tous les nutriments; 2) ces deux phénomènes peuvent être dépassés par la quantité de nutriments mal digérés et 3) les éléments réabsorbés sont mal réutilisés.

\section{SUMmary: Gastrointestinal strongylosis and food absorption.}

The role of malabsorption of nutrients in the mechanism of reduction in productions of the ruminants infested with gastrointestinal nematodes has been a controversial question because of the compensatory absorption and reabsorption in distal part of the small intestine. However, during the last decade, leading research showed that malabsorption has a major role in this mechanism because of 1) compensatory absorption and reabsorption remain limited to some nutrients, 2) large quantities of uncompletely digested nutrients exceeding the intestine capacity, and 3) poor reutilization of reabsorbed nutrients.

\section{INTRODUCTION}

Les strongyloses gastro-intestinales sont reconnues, dans le monde entier, d'être à l'origine de pertes économiques importantes affectant l'élevage des ruminants en général et du mouton en particulier. Si ces pertes peuvent, parfois, être dues aux mortalités, elles sont surtout liées à l'importance de la diminution des productions des animaux qui ne meurent pas de leur parasitisme et qui se manifeste par une baisse du gain de poids (Sykes et Coop, 1976; Dakkak et Ouhelli, 1988), de la production laitière (Thomas et Ali, 1983) ainsi que des performances de la reproduction (Dakkak et Ouhelli, 1988). A ces pertes s'ajoutent celles dues aux modifications de la composition corporelle et tout particulièrement l'augmentation de la teneur en eau et la diminution des graisses et des protéines, ainsi que du calcium et du phosphore au niveau du squelette (Reveron et coll., 1974; Sykes et Coop, 1976). Le rôle de la malabsorption dans la diminution des productions fait l'objet d'une controverse de la part des chercheurs car, en égard aux troubles affectant (1) la perméabilité des muqueuses

Département de Parasitologie et Maladies Parasitaires, Institut Agronomique et Vétérinaire Hassan II, B. P. 6202, Rabat-Instituts, Maroc. gastro-intestinales (Mulligan et coll., 1963; Dakkak et coll., 1985), (2) les sécrétions des hormones (Symons, 1982; Titchen, 1982) et des enzymes (Jones, 1983; Holmes, 1987) intervenant dans la digestion et (3) la motricité du tractus gastro-intestinal (Dakkak, 1984; 1988; Gregory et coll., 1985), le problème de la malabsorption apparaît simple. Il est en réalité très complexe à cause de l'importante capacité du tractus digestif de compenser une malabsorption proximale par une augmentation de l'absorption et une réabsorption des nutriments dans la partie distale. Les données rapportées dans cet article concerneront essentiellement les ruminants.

\section{1 - Apperçu SUR Le DySFonctionnement dU tractus GASTRO-INTESTINAL}

Les troubles de l'absorption des nutriments peuvent être en relation avec ceux affectant la perméabilité des muqueuses infestées, les sécrétions intervenant dans le métabolisme des nutriments et la motricité du tractus gastrointestinal.

Il est actuellement bien établi que les infestations du tractus gastro-intestinal par les nématodes provoquent une augmentation de la perméabilité des muqueuses infestées 
(Mulligan et coll., 1963; Dakkak et coll., 1985; Holmes, 1987). Cette perméabilité anormalement augmentée est à l'origine d'importantes fuites plasmatiques vers la lumière des organes infestés (Mulligan et coll., 1963; Holmes, 1987).

Les infestations de l'abomasum s'accompagnent d'une stimulation de toutes les sécrétions sauf celle de l'acide chlorhydrique qui diminue permettant au $\mathrm{pH}$ du contenu de cet organe d'atteindre la valeur de 6 et même plus (Dakkak et coll., 1985) bien qu'il se produit une importante augmentation de la sécrétion de la gastrine (Titchen, 1982). Les infestations de l'intestin provoquent une augmentation des sécrétions de la cholecystokinine et de la somatostatine (Symons, 1982; Titchen, 1982). Elles entraînent aussi d'importantes modifications de la sécrétion des enzymes des bordures en brosses (ou enzymes de la digestion) avec augmentation des activités de l'acétylcholinestérase, de l'aldolase et de la créatine phosphokinase et diminution de celles de la phosphatase alcaline et de la leucine aminopeptidase (Jones, 1983). Signalons enfin, que ce parasitisme provoque également des troubles affectant les sécrétions (1) des hormones pancréatiques avec particulièrement, diminution de l'insuline, et (2) d'autres hormones dont les corticostéroïdes qui augmentent et la thyroxine qui diminue (Holmes, 1987).

Les troubles affectants la motricité du tractus gastrointestinal sont très importants. En effet, les infestations de l'abomasum provoquent des troubles moteurs affectant non seulement cet organe mais aussi le rumen et l'intestin. Aux niveaux abomasal, duodénal et jéjunal, il se produit une hypermotricité qui s'accompagne d'une augmentation du débit des digesta dans le duodénum et une diminution du temps de transit de ceux-ci dans tout l'intestin (Dakkak, 1984). Cette hypermotricité abomaso-intestinale s'oppose à l'hypomotricité du rumen, du réticulum et de l'omasum (Dakkak, 1988). Contrairement aux infestations de l'abomasum, celles de l'intestin grêle provoquent une inhibition des motricités abomasale et intestinale associée à une diminution du débit abomaso-duodénal et à un ralentissement du transit intestinal (Gregory et coll., 1985).

\section{2 - ÉVOLUTION DES CONNAISSANCES SUR LA MALABSORPTION}

Il ressort des différentes revues bibliographiques consacrées à ce sujet que nos connaissances sur la malabsorption débutent avec les travaux de Stewart (1933) qui font état d'une diminution de la digestion et de l'absorption des protéines chez le mouton soumis à une infestation mixte où prédomine $H$. contortus. Par la suite la malabsorption a été rapportée par de nombreux travaux qui ont démontré qu'elle affecte : 1) les protéines, 2) ce qui est habituellement appelé « énergie »; c'est-à-dire des glucides, des matières grasses et même quelques acides aminés, 3) les minéraux et tout particulièrement le phosphore et le calcium, 4) les vitamines A, C, B6 et B12 et 5), enfin, quelques ions dont particulièrement le sodium. Ces résultats permettent de conclure que la malabsorption des nutriments constitue, avec la diminution de l'appétit, les deux facteurs majeurs du mécanisme de la diminution des productions des animaux infestés. Cependant, avant d'arriver à ces conclusions et à cause, d'une part, des phénomènes d'absorption compensatrice et de réabsorption des nutriments dans la région distale de celle du site de l'infestation et, d'autre part, des différences relevées entre les métabolismes des nutriments absorbés normalement ou réabsorbés (différences que nous étudierons plus loin), nos connaissances sur le sujet sont passées par plusieurs stades.

L'absorption compensatrice dans la région distale au site de l'infestation a été constatée pour la première fois en 1961 chez le rat infesté par Nippostrongylus brasiliensis (Symons, 1961). Elle a suscité par la suite un très grand intérêt de la part des chercheurs tout particulièrement après la démonstration par Mulligan et ses collaborateurs (1963) de l'existence d'importantes fuites plasmatiques au niveau des muqueuses infestées et qui ont fait l'objet de nombreux travaux par la suite dont les résultats ont été rapportés dans la revue bibliographique consacrée par Holmes (1987) à la pathogénie des helminthoses gastro-intestinales. Ces fuites ont été souvent à l'origine d'erreurs dans les bilans qui ne distinguent pas entre les éléments endogènes et les éléments provenant de la ration alimentaire. Les travaux consacrés à la malabsorption vont alors comporter des précisions sur l'importance de l'absorption compensatrice et de la réabsorption. Actuellement, il est bien établi que la malabsorption des nutriments au niveau du site de l'infestation peut, selon la ou les infestation(s) en cause et selon le nutriment, être fortement, peu ou pas compensée dans la région distale.

\section{3 - Malabsorption Des protérnes}

A la suite des découvertes de Symons (1961) et de Mulligan et coll. (1963), de nombreuses études ont montré que, globalement et grâce à l'absorption compensatrice et à la réabsorption, l'absorption des protéines est peu affectée dans la grande majorité des cas. Ces résultats ont incité plusieurs auteurs à conclure que la diminution de la digestion et de l'absorption ne serait pas un facteur majeur dans le mécanisme de la diminution des productions des animaux infestés. Dans le même temps, il a été démontré que cette absorption compensatrice reste limitée dans certains cas. Ainsi, Horak et Clark (1964) rapportent que l'absorption réelle des protéines diminue de $25 \%$ chez le mouton infesté par $O$. circumcincta. De même, selon Roseby et Leng (1974) cette même absorption diminue de 17 à $23 \%$ lorsque le mouton est infesté par $T$. colubriformis. Randall et Gibbs (1981) constatent une diminution de $17 \%$ de l'absorption des matières azotées chez des bovins infestés par O. ostertagi et Cooperia oncophora. Enfin, selon Bremner (1969) l'infestation de l'iléum et du gros intestin par $O$. radiatum provoque chez le bovin une importante 
baisse de l'absorption des nutriments en général qui n'est pas compensée dans la partie distale.

Au cours de la dernière décennie, de nombreuses études ont progressivement montré que l'absorption compensatrice et la réabsorption ont moins d'importance qu'on ne leur avait accordé dans le passé et que les troubles de l'absorption des nutriments avaient des conséquences importantes sur les productions des animaux infestés. En effet, en plus de son incapacité de compenser la malabsorption de certains nutriments comme les vitamines, les minéraux, les sels biliaires, ... etc., dont l'absorption dépend de mécanismes devant se dérouler dans des régions spécialisées de la muqueuse intestinale, l'absorption compensatrice peut être dépassée par la quantité de protéines mal digérées provenant de la ration alimentaire ou des fuites plasmatiques en amont, ou intéresser des acides aminés qui ne peuvent plus être métabolisés convenablement. Dans ce sens, il a été démontré que dans les infestations de l'abomasum, 20 $\%$ des protéines provenant des fuites plasmatiques ne sont pas réabsorbés (Symons, 1976). De même, au cours de l'infestation de l'intestin du mouton par $T$. colubriformis, Roseby et Leng (1974), constatant une importante augmentation de l'entrée des matières azotées dans le cæcum accompagnée d'une augmentation de la fermentation dans cet organe, concluent que l'absorption compensatrice et la réabsorption des protéines restent limitées. Plus tard, il a été démontré qu'au cours de cette même infestation $30 \%$ des protéines provenant des fuites plasmatiques ne sont pas réabsorbés (Holmes, 1987). En accord avec ces résultats, Symons (1982) pense qu'il existe chez le mouton une relation étroite entre les fuites plasmatiques et les diminutions des productions et confirment ainsi les conclusions de Roseby et Leng (1974).

D'autre part, les troubles affectant les processus de la fermentation des digesta dans le rumen (Rowe et coll., 1988) provoquent une augmentation des débits abomaso-duodénal et duodéno-iléal de l'azote non ammoniacal. Cette augmentation peut être à l'origine de celle de la population des bactéries dans les différentes parties de l'intestin qui a été démontrée par Jennings et coll. (1966). Ces modifications peuvent entraîner, d'une part, une diminution des acides aminés à absorber particulièrement aggravée par la déamination de ces acides qui a été constatée par Roseby et Leng (1974) chez le mouton infesté par T. colubriformis et, d'autre part, une augmentation de la production des acides gras volatiles dans le gros intestin qui ne peuvent plus être utilisés par l'organisme.

Enfin, la diminution des acides aminés à absorber et les fuites plasmatiques ainsi que les modifications du métabolisme des protéines intestinales (augmentation du renouvellement des cellules épithéliales, de la production de mucus et de l'absorption compensatrice) sont à l'origine d'importants troubles du métabolisme des protéines chez les animaux infestés. Il a été démontré dans ce sens que l'effica- cité de la réutilisation des acides aminés réabsorbés dans la synthèse des protéines dans les différentes parties du corps est faible comme le montrent l'augmentation de la synthèse de l'urée, de son excrétion et de son recyclage (Roseby et Leng, 1974; Rowe et coll., 1988). Selon Holmes (1987), les protéines réabsorbées subissent une déamination dans le foie puis l'azote est excrété sous forme d'urée. Les fuites plasmatiques et cette mauvaise réutilisation des protéines réabsorbées représentent deux facteurs majeurs dans le mécanisme des diminutions des productions chez les animaux infestés. En effet, dans le but de compenser les fuites plasmatiques et maintenir l'homéostasie, il se produit une augmentation de la synthèse des protéines plasmatiques (essentiellement de l'albumine) par le foie (Symons, 1976; Coop et coll., 1982). Les protéines réabsorbées étant faiblement utilisées pour cette synthèse, il se produit une augmentation du catabolisme des protéines des muscles, de la moelle osseuse, de la laine, de la peau... etc. (Symons, 1976; 1982; Holmes, 1987) pour satisfaire l'augmentation de la demande métabolique.

\section{4 - Malabsorption des aUtres nUtriments}

Par rapport aux troubles de l'absorption des protéines, ceux affectant l'absorption des autres nutriments n'ont pas fait l'objet d'études aussi approfondies.

De nombreuses études ont montré que la diminution de l'appétit, pouvant atteindre $30 \%$ dans les infestations de l'abomasum par $O$. circumcincta et jusqu'à $50 \%$ dans celles de l'intestin, est un facteur majeur qui affecte, chez les ruminants infestés, la disponibilité de ce qui est habituellement appelé énergie. Cependant, il a été démontré qu'à cette diminution de l'apport s'ajoute celle de l'efficacité de l'utilisation de l'énergie ingérée atteignant $30 \%$ dans l'infestation de l'abomasum par $O$. circumcincta (Coop et coll., 1982) et 42 à $50 \%$ dans celle de l'intestin par T. colubriformis (Sykes et Coop, 1976). L'augmentation de l'élimination fécale de l'énergie constatée chez les animaux infestés indique sa malabsorption et serait une des raisons de la diminution de l'efficacité de son utilisation. Enfin, signalons que la malabsorption du glucose a été démontrée chez le rat infesté par Nippostrongylus brasiliensis ou par Trichinella spiralis (Symons, 1982).

L'absorption et la réabsorption compensatrice éventuelle des minéraux n'ont pas encore été bien étudiées. Des recherches récentes ont montré que les troubles phosphocalciques sont importants et provoquent une diminution de la croissance et de la minéralisation du squelette chez le mouton infesté par $O$. circumcincta (Coop et coll., 1982), T. colubriformis (Sykes et Coop, 1976) et chez le bovin infesté par des trichostrongylidés (Enterocasso et coll., 1986). La diminution de l'absorption du phosphore rapportée par Reveron et coll. dès 1974 a été confirmée par Wilson et Field (1983) qui ont démontré en plus la diminution de son recyclage. Des résultats similaires ont été 
obtenus par Brown et coll. (1987) dans les infestations du mouton par $O$. circumcincta ou par $T$. colubriformis et qui ont précisé en plus que l'absorption compensatrice du phosphore et sa réabsorption sont très limitées. En ce qui concerne le calcium, dès 1969, Waymarck et Torbert avaient constaté une légère augmentation de son excrétion fécale chez le bovin infesté par Ostertagia spp., que Wilson et Field (1983) retrouvent chez le mouton infesté par $O$. circumcincta ou T. colubriformis. Selon Brown et coll. (1987), il se produit une importante absorption compensatrice du calcium dans le gros intestin des animaux infestés par ces mêmes nématodes. Cependant, pour ces mêmes auteurs, la malabsorption du calcium existe puisque les animaux présentent la coexistence d'une hypophosphatémie et d'une hypocalcémie. D'autre part, il a été constaté que l'absorption du magnésium ne semble pas être affectée par les strongyloses gastro-intestinales (Brown et coll., 1987), alors que celle du fer augmente dans l'infestation de l'abomasum par H. contortus (Holmes, 1987). Enfin, signalons que quelques études (voir Downey, 1966) ont montré que les nématodoses gastro-intestinales affectent aussi l'absorption des vitamines $\mathrm{A}, \mathrm{C}, \mathrm{B} 6$ et $\mathrm{B} 12$ dont les concentrations plasmatiques diminuent et que, selon Ross et coll. (1970), l'absorption du sodium diminue chez le bovin infesté par $T$. axei.

\section{RÉFÉRENCES}

Brown M. D., Poppi D. P., Sykes A. R. : Mineral transactions along the digestive tract of lambs exposed to mixed nematode infection (Trichostrongylus colubriformis and Ostertagia circumcincta). In Nuclear techniques in the studies and control of parasitic diseases of livestock. International Atomic Energy Agency, Vienna, 1987, 149-156.

Bremner K. C. : Pathogenic factors in experimental bovine œsophagostromosis. IV. Exudative enteropathy as a cause of hypoproteinaemia. Exp. Parasitol., 1969, 25, 382-394.

Coop R. L., Sykes A. R., Angus K. W. : The effects of three levels of intake of Ostertagia circumcincta larvae on grouth rate, food intak and body composition of growing lamb. J. Agric. Sci., 1982, 98, 247-255.

Dakkak A. : Physiopathologie digestive de l'haemonchose et de l'ostertagiose ovines: Interaction hôte-parasites. Thèse Doct. es Sci. Nat. Institut National Polytechnique de Toulouse, 1984.

Dakkak A. : Ovine haemonchosis: Digestive pathophysiology and vaccination trials. In Nuclear and related techniques in animal production and health. International Atomic Energy Agency, Vienna, 1988, 365-380.

Dakkak A., Ouhelli H. : Impact on productivity and epidemiology of gastrointestinal and lungworm parasites in sheep in Morocco. In: Thomson E. F. et Thomson F. S. (eds.). Increasing small ruminant productivity in semi-arid areas. Kluwer Academic Publishers, Dordrecht, Netherlands, 1988, 243-255.

Dakkak A., Daoudi A., Ruckebusch Y. : Haemonchus contortus et Ostertagia circumcincta: Fenbendazole treatment and abomasol permeability changes in sheep. Am. J. Vet. Res., 1985, 46, 209-211.
Downey N. E. : Some relationships between Trichostrongylid infestation and cobalt status in lambs. III: Trichostrongylus axei and Ostertagia circumcincta infestation. Br. Vet. J., 1966, 122, 316-324.

Enterocasso C., Parkins J. J., Armour J., Bairden K., Mc William P. N. : Métabolisme and grouth studies in housed calves given a morantel sustained release bolus and exposed to natural trichostrongyle infection. Res. Vet. Sci., 1986, 40, 65-75.

Gregory P. C., Wenham G., Poppi D., Coop R. L., Mac Rae J. C., Miller S. J. : The influence of chronic subclinical infection of Trichostrongylus Colubriformis on gastrointestinal motility and digesta flow in sheep. Parasitology, 1985, 91, 381-396.

Holmes P. H. : Physiology of parasitic infections. Parasitology, 1987, 94, S29-S51.

Horak I. G., Clark R. : The pathological physiology of Ostertagia circumcincta Onderstepoort. J. Vet. Res., 1964, 31, 163-176.

Jennings F. W., Armour J., Lawson D. D., Roberts R. : Experimental Ostertagia ostertagi infections in calves: studies with abomasal cannulas. Am. J. Vet. Res., 1966, 27, 1249-1257.

Jones D. D. : Intestinal enzyme activity in lambs chronically infected with Trichostrongylus colubriformis: Effect of anthelminthic treatment. Vet. Parasitol., 1983, 12, 79-89.

Mulligan W., Dalton R. G., Anderson N. : Ostertagiasis in cattle. Vet. Rec., 1963, 75, 1014-1016.

Randall R. W., Gibbs H. C. : Effect of clinical and subclinical gastrointestinal helminthiasis on digestion and energy metabolism in calves. Am. J. Vet. Res., 1981, 42, 1730-1734.

Reveron A. E., Topps J. H., Gelman A. L. : Mineral metabolism and squeletal development of lambs affected by Trichostrongylus colubriformis. Res. Vet. Sci., 1974, 16, 310-319.

Roseby F. B., Leng R. A. : Effect of Trichostrongylus colubriformis (Nematoda) on the nutrition and metabolism of sheep. II. Metabolism of urea. Aust. J. Agric. Res., 1974, 25, 363-367.

Ross J. G., Todd J. R., Purcell D. A. : Nutritional balance trials with calves experimentally infected with Trichostrongylus axei. Br. Vet. J., 1970, 126, 393-400.

Rowe J. B., Nolan J. V., de Channeet G., Teleni E. : The effect of haemonchosis and blood loss into the abomasum on digestion in sheep. Br. J. Nutr., 1988, 59, 125-139.

Sykes A. R., Coop R. : Intake and utilisation of food by growing lambs with parasitic damage to the small intestine caused by daily dosing with Trichostrongylus colubriformis larvae. J. Agric. Sci., 1976, 86, 507-515.

Symons L. E. A. : Pathology of infestation of the rate with Nippostrongylus muris. VI. Absorption in vivo from the distal ileum. Am. J. Biol. Sci., 1961, 14, 163-171.

Symons L. E. A. : Malabsorption. In: Soulsby E. J. L. (Ed.). Pathophysiology of parasitic infection. Academic Press, New York, 1976, 11-21.

Symons L. E. A. : Gastrointestinal pathology induced by enteric parasites. In: Mettrick D. F. et Desser S. S. (Eds): Parasites, their world and ours. Elsevier, Amsterdam, 1982.

Titchen D. A. : The role hormones in the reaction of the host to enteric parasites. In: Mettrick D. F. et Desser S. S. (Eds.): Parasites, their world and ours. Elsevier, Amsterdam, 1982, 244-245.

Thomas R. J., Ali D. A. : The effect of Haemonchus contortus infection on the pregnant and lactating awe. Int. J. Parasitol., 1983, 13, 393-398.

Wilson W. D., Field A. C. : Absorption and secretion of calcium and phosphorus in the alimentary tract of lambs infected with daily doses of Trichostrongylus colubriformis or Ostertagia circumcincta larvae. J. Comp. Pathol., 1983, 93, 61-71. 